\title{
HIGH POWER MITER-BEND FOR THE NEXT LINEAR COLLIDER
}

\author{
M.A.Shapiro and R.J.Temkin, Plasma Science and Fusion Center, \\ Massachusetts Institute of Technology, Cambridge MA 02139
}

\begin{abstract}
In the design of the Next Linear Collider (NLC), it may be necessary to transmit very high power levels (hundreds of megawatts) of microwave radiation over distances of tens of meters. The microwave transmission system will require the use of oversized waveguides and higher order modes in order to either avoid breakdown or to optimize the microwave system properties. We have studied one critical component of the proposed waveguide transmission system, namely a miter- bend for the $\mathrm{TE}_{01}$ mode capable of transmitting over $600 \mathrm{MW}$ of power at $11.424 \mathrm{GHz}$. The miter-bend is a quasi-optical 90-degree bend. The design is based on previous, successful designs of miter-bends for high average power gyrotrons at frequencies of $35 \mathrm{GHz}$ and above. The design consists of a profiled mirror and two oversized rippled-wall mode converters optimized to provide less than $0.1 \mathrm{~dB}$ operating mode losses.
\end{abstract}

\section{INTRODUCTION}

In the NLC Test Accelerator built at SLAC, the pulse compressor SLED-II producing $200 \mathrm{MW}, 250 \mathrm{~ns}, 11.424$ $\mathrm{GHz}$ pulses has been designed and successfully tested [1]. This pulse compressor contains the following microwave components capable of handling $60 \mathrm{MW}$ pulses: circular waveguides operating at the $\mathrm{TE}_{01}$ mode, a magic tee, and a flower-petal $\mathrm{TE}_{10}$ to $\mathrm{TE}_{01}$ mode transducer [2]. To make a 90-degree bend of the circular waveguide $\mathrm{TE}_{01}$ mode transmission line, two flower-petal transducers and the rectangular waveguide bend are used. To operate at high powers, a bend of a ridged circular waveguide of a $4 \mathrm{~cm}$ diameter has been proposed [3]. However, such a design cannot be used with a $12 \mathrm{~cm}$ diameter waveguide because of sufficient conversion of the $\mathrm{TE}_{01}$ mode into spurious modes and, as a result, breakdowns.

The Delay Line Distribution System (DLDS) as a pulse compressor for NLC is under design at SLAC to enhance the peak power up to $600 \mathrm{MW}$ [4]. We propose a quasi-optical miter-bend which can be used with a $12.7 \mathrm{~cm}$ circular waveguide in DLDS. This design is based on the developments of miter-bends for the $35 \mathrm{GHz} \mathrm{TE}_{01}$ mode circular waveguides [5], and for the $110 \mathrm{GHz} \mathrm{HE}_{11}$ mode corrugated waveguides [6] employed to transmit to long distances the radiation of high power gyrotrons.

\section{MITER-BEND DESCRIPTION}

In the quasi-optical miter-bend, we employ two circular waveguide sections cut under the angle of $45^{\circ}$ and placed such that the waveguide axes form an angle of $90^{\circ}$. These waveguides are optically connected using a mirror (Fig.1). The waveguide wall goes all the way to the mirror. This eliminates diffraction expansion of the mode in the direction perpendicular to Fig.1. The mirror is profiled to compensate for the diffraction in the plane of Fig.1.

An unusual feature of the proposed miter-bend is that the operating mode is a mixture of waveguide modes. It is known that the diffraction of the $\mathrm{TE}_{01}$ mode at an open end of the waveguide is small compared to that the other modes since the electric field is zero at the wall. Nevetheless, the mixture of the $\mathrm{TE}_{01}$ and $\mathrm{TE}_{02}$ modes forms not only the zero field but the derivative of the electric field equal to zero at the junction of the waveguide and the mirror. This allows to reduce diffraction $[5,6]$. By this reason, we introduce two periodic rippled-wall waveguides (mode converters) converting the $\mathrm{TE}_{01}$ mode into the optimum mixture of the $\mathrm{TE}_{01}$ and $\mathrm{TE}_{02}$ modes (Fig1.).

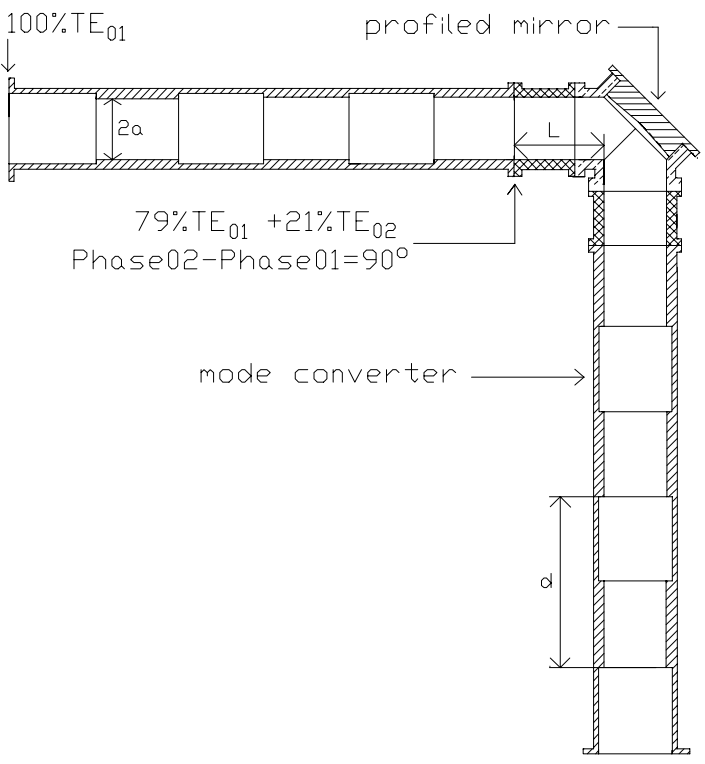

Fig.1. Miter-bend schematic. 


\section{MITER-BEND DESIGN}

We design the miter-bend for the waveguide of the radius $a=6.35 \mathrm{~cm}$, which is oversized at the frequency of 11.424 GHz. Therefore, a quasi-optical approach, valid if $k a>>1(k$ is a wavenumber), can be employed for calculations. Within this approximation, the mirror is represented as a thin phase corrector. The equivalent quasi-optical schematic includes the waveguide cut by two $45^{\circ}$ planes and the phase corrector placed as shown in Fig.2.

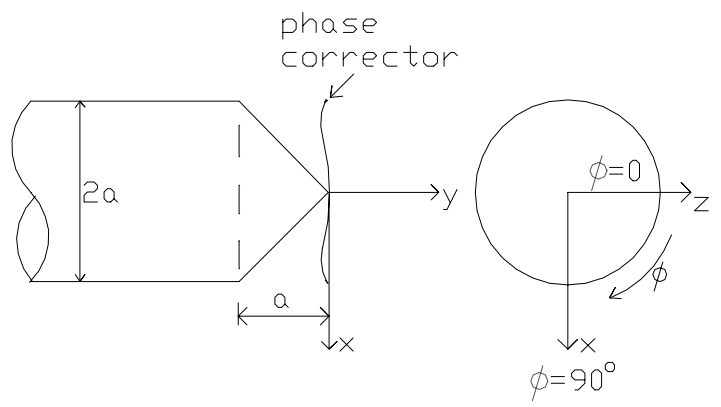

Fig.2. Quasi-optical schematic.

\subsection{Diffraction Losses}

The procedure of numerical calculation is the following. We take an initial field distribution as a superposition of the $\mathrm{TE}_{01}$ and $\mathrm{TE}_{02}$ modes and propagate it through the gap of the length of $a$ to the plane of the phase corrector. In the direction $\phi=0$ (Fig.2) at the corrector, the incident electric field is the waveguide field distribution over radius $r$ :

$$
E_{\phi=0}^{i n c}=\sum_{n=1,2} \frac{A_{0 n}}{\sqrt{R_{0 n}}} \frac{J_{1}\left(X_{0 n} r / a\right)}{a J_{0}\left(X_{0 n}\right)} \exp \left(-j k R_{0 n} a\right)
$$

where $A_{01}$ and $A_{02}$ are amplitudes of the $\mathrm{TE}_{01}$ and $\mathrm{TE}_{02}$ modes, $X_{01}=3.832, X_{02}=7.016$, and

$$
R_{0 n}=\sqrt{1-\left(X_{0 n} / k a\right)^{2}}
$$

In the perpendicular direction $\phi=90^{\circ}$ (Fig.2), the incident electric field distribution varies due to the diffraction and is expressed as follows [6]:

$$
\begin{aligned}
E_{\phi=90}^{i n c}= & \sum_{n=1,2} \frac{A_{0 n}}{\sqrt{R_{0 n}}} X_{0 n} \times \\
& \int_{0}^{\infty} \frac{\gamma a J_{1}(\gamma a) J_{1}(\gamma r)}{(\gamma a)^{2}-X_{0 n}^{2}} \exp \left(-j \sqrt{k^{2}-\gamma^{2}} a\right) d \gamma
\end{aligned}
$$

To calculate the diffraction losses we represent the latter field distribution as a mode series of the waveguide of the radius $a$, propagate it back at the distance of $a$ using the same integral transformation, and again express the result as a superposition of the $\mathrm{TE}_{\mathrm{on}}$ modes. The relationship between the initial amplitudes $A^{\text {ini }}$ and the receiving amplitudes $A^{\text {rec }}$ gives the efficiency of the miter-bend:

$$
\eta=\frac{1}{2}+\frac{1}{2} \frac{\left|A_{01}^{r e c}\right|^{2}+\left|A_{02}^{r e c}\right|^{2}}{\left|A_{01}^{i n i}\right|^{2}+\left|A_{02}^{i n i}\right|^{2}}
$$

Here we use the fact that a half area of the gap is covered by the waveguide wall (Fig.2), so the diffraction losses are twice smaller than those for the uncovered gap.

\subsection{Mode Converter}

We specify the amplitudes $A_{01}$ of $\mathrm{TE}_{01}$ mode and $A_{02}$ of $\mathrm{TE}_{02}$ mode to minimize the diffraction at the mirror aperture. A smaller diffraction at the mirror can be obtained if $(d / d r) E^{i n c}=0$ at the $r=a$, at the corrector (Fig.2). This gives the amplitude ratio $A_{02} / A_{01}=$ $\left(R_{02} / R_{01}\right)^{1 / 2} X_{01} / X_{02}=-0.51$ at the corrector, and $A_{02} / A_{01}=$ $0.51 \exp (j \alpha)$ at the distance of $a$ from the mirror, where $\alpha=\pi-k\left(R_{01}-R_{02}\right) a=110^{\circ}$.

This amplitude ratio corresponds to $79 \%$ of power in the $\mathrm{TE}_{01}$ mode and $21 \%$ of power in the $\mathrm{TE}_{02}$ mode. To convert the pure $\mathrm{TE}_{01}$ mode into this mixture, the periodic waveguide section (Fig.1) of the following geometry can be used: period $d=2 \pi /\left(k\left(R_{01}-R_{02}\right)\right)=32.6$ $\mathrm{cm}$, total length $M d$, where $M \geq 3$ is the number of periods, the step of the waveguide radius is

$$
\varepsilon=\frac{a}{M} \frac{X_{02}^{2}-X_{01}^{2}}{X_{01} X_{02}} \frac{\sqrt{R_{01} R_{02}}}{R_{01}+R_{02}} \frac{\arctan 0.51}{\pi}
$$

Thus, for $M=3, \varepsilon=0.2 \mathrm{~cm}$.

The phase of the $\mathrm{TE}_{02}$ mode is of $90^{\circ}$ ahead of the phase of the $\mathrm{TE}_{01}$ mode at the output of the mode converter. Therefore, to get these modes in phase at the mirror, we insert a waveguide section such that the length between the mode converter and the mirror is $L=0.75 d$ (Fig.1).

\subsection{Mirror Profile and Efficiency}

The incident electric field at the corrector can be written in coordinates $(x, z)$ of Fig. 2 as follows:

$E^{i n c}(x, z)=\frac{1}{2}\left(E_{\phi=0}^{i n c}+E_{\phi=90}^{i n c}\right)+\frac{1}{2}\left(E_{\phi=0}^{i n c}-E_{\phi=90}^{i n c}\right) \cos \left(2 \arctan \frac{x}{z}\right)$

To calculate the mirror profile we determine the phase distribution of this field:

$$
\Phi^{i n c}(x, z)=\arg \left(E^{i n c}\right)
$$

We represent the mirror surface in the coordinates $\left(x_{s}, y_{s}\right)$, connecting to the surface. Since the mirror is titled by $45^{\circ}$, the relationship between the coordinates $\left(x, y_{s}\right)$ and $(x, y)$ is $y=y_{s} / \cos 45^{\circ}$ and $x=x_{s} \cos 45^{\circ}$. Therefore, the profile of the mirror, to conjugate the phase, is the following: 


$$
y_{s}=\Delta\left(x_{s}, z\right)=-2 \cos 45^{\circ} \Phi^{i n c}\left(x_{s} \cos 45^{\circ}, z\right) / k
$$

For the mode amplitude ratio of 0.51 and the mode phase shift $\alpha=110^{\circ}$ determined in Sec.3.2, the numerically calculated efficiency of the miter-bend is $\eta=0.983$, which corresponds to less than $0.1 \mathrm{~dB}$ diffraction losses. The 3D mirror profile is depicted in Fig.3. The mirror is almost cylindrical because there is no diffraction in $z$-direction (Fig.2), plus the phase shift between the modes is specified to provide a flat phase front in $z$-direction at the mirror. A correction in $x$-direction is needed, and the optimum profile is plotted in Fig.4.

The simulations indicated that the efficiency varies from 0.984 to 0.980 while the ratio of mode amplitudes $A_{02} / A_{01}$ varies from 0.4 to 0.6 and the phase shift $\alpha=110^{\circ}$ remains the same. In this case, the mirror profile is cylindrical, and the losses are only due to the diffraction at the waveguide aperture.

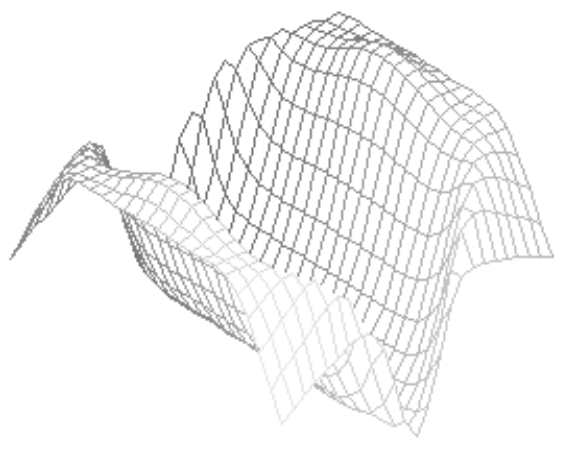

Fig.3. 3D profile of the mirror (off scale).

The efficiency can be raised up to 0.993 using optimization by $\alpha$. Varying $\alpha$, we minimize the diffraction at the waveguide aperture as well as at the mirror. However, there is a parasitic polarization conversion due to scattering at a non-cylindrical mirror. This effect has not been taken into account by the quasi-optical approach. The estimation indicates that the polarization losses are about 0.013 , which brings the efficiency down to 0.98 .

\section{CONCLUSIONS}

We design a quasi-optical miter-bend for DLDS. It is capable to handle high peak power microwave pulses because highly ovesized waveguides are utilized. Such a design cannot be employed with a small diameter $(4 \mathrm{~cm})$ waveguide, smooth bends are preferable for that.

The efficiency of the miter-bend meets the DLDS requirements. Less than $0.1 \mathrm{~dB}$ losses are obtained in the calculations. The mirror can be made mechanically steerable to reach maximum efficiency in a cold test. The length $L$ of the straight waveguide section can be optimized in a cold test as well. The mode converter is designed as a periodic waveguide of the length of 3 periods, and the corrugation is rectangular.
The mode converter can be modified to employ a sinusoidal corrugation. The length of the mode converter can be lowered, though the corrugation profile should be optimized for that.

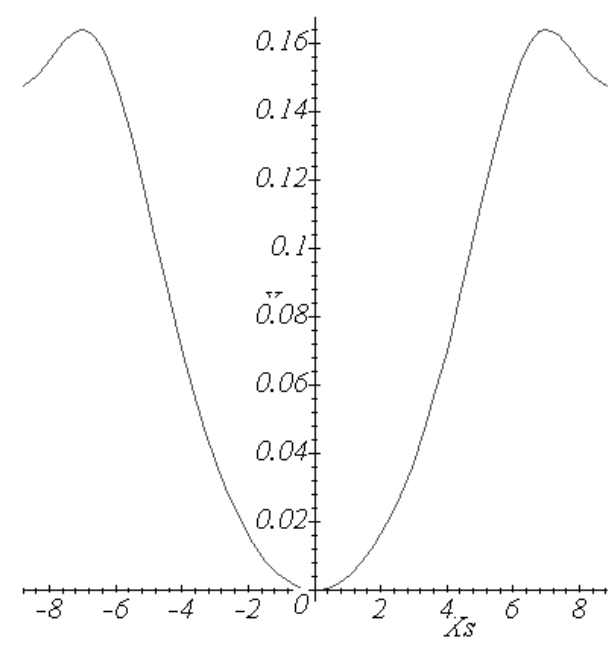

Fig.4. Mirror cut in $\mathrm{x}$-direction (dimensions in $\mathrm{cm}$ ).

\section{ACKNOWLEDGMENTS}

We are thankful to Dr. Sami Tantawi for useful discussions. This research was supported by US Department of Energy, High Energy Physics.

\section{REFERENCES}

[1] S.G.Tantawi et al., 'The Next Linear Collider Test Accelerator's RF Pulse Compressors and Transmission Systems', Proc. of 1997 Particle Accelerator Conference, IEEE, p.3192.

[2] S.G.Tantawi, K.Ko, and N.Kroll, 'Numerical Design and Analysis of a Compact TE10 to TE01 Mode Transducer', Conference on Computational Accelerator Physics, Los Alamos, NM, 1993, AIP Conference Proceedings 297, p.99.

[3] C.Nantista, N.M.Kroll, and E.M.Nelson, 'Design of a $90^{\circ}$ Overmoded Waveguide Bend', Proc. of 1993 Particle Accelerator Conference, IEEE, p.983.

[4] S.G.Tantawi et al., 'A Multi-Moded RF Delay Line Distribution System for the Next Linear Collider', Proc. of 1998 European Particle Accelerator Conference, p.305.

[5] M.A.Shapiro and S.N.Vlasov, 'Study of Combined Transmission Line for High Power Wave Generated by a Gyrotron in the Millimetre Wavelength Range', International Journal of Electronics, 1992, Vol.72, p.1127.

[6] J.L.Doane and C.P.Moeller, 'HE11 Mitre Bends and Gaps in a Circular Corrugated Waveguide', International Journal of Electronics, 1994, vol.77, p.489. 